\title{
Retraction Note to: Improved Endovascular Coiling of Wide-Neck Intracranial Aneurysms in Elderly Patients by Double-Microcatheter Technique
}

\author{
Xiang $\mathrm{Xu}^{1} \cdot \mathrm{Yu} \mathrm{Zheng}^{1} \cdot$ Dayong Wang ${ }^{1} \cdot$ Jianzhong Cui ${ }^{1} \cdot \mathrm{Xiaoming} \mathrm{Shang}^{2}$
}

Accepted: 7 January 2022 / Published online: 22 January 2022

(c) Springer Science+Business Media, LLC, part of Springer Nature 2022

Retraction Note to: Cell Biochem Biophys (2015) 71:1281-1286, https://doi.org/10.1007/s12013-014-0344-3, published online 05 Dec 2014

The Editor in Chief has retracted this article because it contains material that substantially overlaps with the following article [1]. The editor and publisher were not able to obtain current email addresses for Xiang Xu, Yu Zheng, Dayong Wang, Jianzhong Cui and Xiaoming Shang.

\section{Reference}

1. Lee, J. Y. et al. (2011). Endovascular treatment of wide-neck intracranial aneurysms using a microcatheter protective technique: results and outcomes in 75 aneurysms. AJNR American Journal of Neuroradiology, 32, 917-22.

The original article can be found online at https://doi.org/10.1007/ s12013-014-0344-3.

Xiang Xu

drxuxiang@163.com

1 Department of Neurosurgery, Tangshan Gongren Hospital, Tangshan 063000 Hebei, China

2 Department of Cardiology, Tangshan Gongren Hospital, Tangshan 063000 Hebei, China 